\title{
Aggregated Stochastic State Classes in Quantitative Evaluation of non-Markovian Stochastic Petri Nets
}

\author{
András Horváth, Enrico Vicario \\ Dipartimento di Informatica - Università di Torino - horvath@di.unito.it \\ Dipartimento Sistemi e Informatica - Università di Firenze - vicario@dsi.unifi.it
}

\begin{abstract}
The method of stochastic state classes provides a new approach for the analysis of non-Markovian stochastic Petri Nets, which relies on the stochastic expansion of the graph of nondeterministic state classes based on Difference Bounds Matrix (DBM) which is usually employed in qualitative verification. In so doing, the method is able to manage multiple concurrent nonexponential (GEN) transitions and largely extends the class of models that are amenable to quantitative evaluation. However, its application requires that every cycle in the graph of nondeterministic state classes visits at least a regeneration point where all GEN transitions are newly enabled. In particular, this rules out models whose non-deterministic class graph includes cycles within a Continuous Time Markov Chain (CTMC) subordinated to the activity period of one or more GEN transitions.

In this paper, we propose an extension that overcomes this limitation by aggregating together classes that are reached through firings that do not change the enabling status of GEN transitions. This enlarges the class of models that can be analysed through the method of stochastic state classes and makes it become a proper extension of the class of models that satisfies the so called enabling restriction.
\end{abstract}

Index Terms-non-Markovian stochastic Petri nets, stochastic time Petri nets, steady state analysis, stochastic state classes.

\section{INTRODUCTION}

Non-Markovian stochastic Petri nets enable representation of models with timings characterised by general (GEN) probability distributions, possibly supported over finite domains. This captures the behaviour of systems that accumulate memory of elapsed time, as often occurring in timed communication protocols, real time scheduling and various other relevant application contexts. The capability to express memory is paid in terms of feasibility and complexity of the analysis, which motivated the development of a large literature. In particular, results concerning densely timed models were consolidated around a few major techniques [1], [2], [3], [4], [5].

In the theory of supplementary variables [1], [6], the logical state is supplemented by the vector of ages of the enabled general transitions, and its evolution is described through a set of integro-differential state equations. Even if formulated for any number of concurrent generally distributed transitions, practical tractability requires that two generally distributed transitions are not enabled simultaneously. The same enabling restriction is applied in [2], [3], to guarantee that the stochastic process behaves either as a continuous time Markovian chain (CTMC) or as a CTMC subordinated to the activity period of a generally distributed transition. An embedded discrete time Markov chain (DTMC) is obtained by sampling the process after any firing with no persistent general transition, i.e. at regeneration points where the age of general transitions is null, thus enabling application of Markov Renewal Theory. The limit of the enabling restriction is overcome in [4], [5] under the assumption that generally distributed transitions are deterministic. The process is observed through a synchronous sampling at equidistant time points yielding a general state space Markov chain (GSSMC), whose kernel of transition probabilities at subsequent time points is derived through numerical integration.

More recently, [7], [8], [9] proposed a new technique for quantitative evaluation of models with multiple concurrent generally distributed timers, that extends state space analysis of densely timed models [10], [11], [12] with symbolic derivation of a probability distribution over DBM domains. Following this approach, the graph of non-deterministic state classes usually employed in a variety of verification tools [13], [14], [15], [16] can be expanded into a stochastic class graph that makes explicit transition probabilities among classes and probability distributions of the sojourn time in each class. This puts a higher effort in the construction of the graph-theoretical abstraction of the process under analysis, but circumvents the complexity in the solution of integro-differential equations that support the evaluation.

The theory of stochastic state classes is able to manage models with multiple concurrent non-deterministic generally distributed timers with possibly bounded support. This includes a large class of models that were not tractable with any previous technique, either for structural restrictions [2], [3], [4], [5], or for practical reasons [1]. However, due to the need to enumerate stochastic states observed after every transition firing, the theory is limited to the class of models for which any cycle in the state space visits at least one regeneration class where GEN transitions are either disabled or newly enabled.

In this paper, we combine the approach of stochastic state classes with concepts of the literature of non-Markovian Stochastic Petri Nets, and in particular with the concept of CTMC subordinated to the period of activity of GEN transitions. Specifically, we propose a technique that aggregates together stochastic classes that are visited without changing the enabling status of GEN transitions. This relaxes the conditions that guarantee bounded enumeration so as to encompass models with cycles that do not change the enabling status of one or more GEN transitions with possibly different enabling and disabling/firing times. The resulting technique can be applied to any model that is amenable to analysis through Markov 
Renewal Theory [2], [3] or through the method of (single) supplementary variable [1] or through the theory of stochastic state classes [7], [9] and extends each of these classes.

The rest of the paper is organised as follows. In Sect.II, we recall syntax and semantics of a class of non-Markovian stochastic Petri Nets. In Sect.III, we introduce a simple but non-easy case that illustrates the limits of applicability of existing techniques, and we then introduce the concept of aggregation in the space of stochastic state classes. In Sect.IV, we develop the calculus for the enumeration of aggregated stochastic classes, we demonstrate its termination under conditions that extend the theory of stochastic state classes, and we show how the graph can be used to solve steady state analysis. The overall development is illustrated in Sect.V and conclusions are drawn in Sect.VI.

\section{Stochastic Time Petri Nets}

We recall here syntax and semantics of a class of nonMarkovian stochastic Petri nets, which we call stochastic Time Petri Net (sTPN) [7], [17], [9]:

$$
s T P N=\left\langle P ; T ; A^{-} ; A^{+} ; m_{0} ; E F T ; L F T ; \tau_{0} ; \mathcal{F} ; \mathcal{C}\right\rangle
$$

As in Time Petri Nets [18], [11], [10]: $P$ is a set of places; $T$ a set of transitions disjoint from $P ; A^{-} \subseteq P \times T$ and $A^{+} \subseteq T \times P$ are pre- and post-conditions; $m_{0}: P \rightarrow \mathbb{N}$ is the (initial) marking. $\tau_{0}$ associates each transition $t \in T$ with an (initial) time to fire $\left(\tau_{0}: T \rightarrow \mathbb{R}_{0}^{+}\right.$, where $\mathbb{R}_{0}^{+}$denotes the set of non-negative real numbers); besides, EFT $: T \rightarrow \mathbb{R}_{0}^{+}$ and $L F T: T \rightarrow \mathbb{R}_{0}^{+} \cup\{+\infty\}$ associate each transition with an Earliest and a Latest Firing Time, with $E F T(t) \leq L F T(t)$.

In addition: $\mathcal{C}$ associates each transition with a real-valued weight; and $\mathcal{F}$ associates each transition $t \in T$ with a probability distribution $F_{t}()$ supported in the firing interval $[\operatorname{EFT}(t), \operatorname{LFT}(t)]$.

The state of an sTPN is a pair $s=\langle m, \tau\rangle$, where $m: P \rightarrow$ $\mathbb{N}$ is a marking and $\tau: T \rightarrow \mathbb{R}_{0}^{+}$associates each transition with a time to fire.

A transition $t_{o}$ is enabled if each of its input places contains at least one token, and it is firable if it is enabled and its time to fire $\tau\left(t_{o}\right)$ is not higher than that of any other enabled transition. When multiple transitions are firable, the choice is resolved through a random switch determined by the weight $\mathcal{C}$ as follows: $\operatorname{Prob}\left\{t_{0}\right.$ is selected $\}=\frac{\mathcal{C}\left(t_{o}\right)}{\sum_{t_{i} \in T^{f}(s)} \mathcal{C}\left(t_{i}\right)}$, where $T^{f}(s)$ is the set of transitions that are firable in state $s$.

When a transition $t_{o}$ fires, the state $s=\langle m, \tau\rangle$ is replaced by $s^{\prime}=\left\langle m^{\prime}, \tau^{\prime}\right\rangle$, which we write as $s \stackrel{t_{o}}{\rightarrow} s^{\prime}$. Marking $m^{\prime}$ is derived from $m$ by removing a token from each input place of $t_{o}$, and by adding a token to each output place of $t_{o}$ :

$$
\begin{array}{ll}
m_{t m p}(p)=m(p)-1 & \forall p .\left\langle p, t_{o}\right\rangle \in A^{-} \\
m^{\prime}(p)=m_{t m p}(p)+1 & \forall p .\left\langle t_{o}, p\right\rangle \in A^{+}
\end{array}
$$

Transitions that are enabled both by the intermediate marking $m_{t m p}$ and by $m^{\prime}$ are said persistent, while those that are enabled by $m^{\prime}$ but not by $m_{t m p}$ are said newly enabled. If $t_{o}$ is still enabled after its own firing, it is always regarded as newly enabled [11], [10]. For any transition $t_{i}$ that is persistent after the firing of $t_{o}$, the time to fire is reduced by the time elapsed in the previous state: $\tau^{\prime}\left(t_{i}\right)=\tau\left(t_{i}\right)-\tau\left(t_{o}\right)$. Whereas, for any transition $t_{a}$ that is newly enabled after the firing of $t_{o}$, the time to fire takes a random value sampled in the firing interval according to the probability distribution $F_{t_{a}}()$ :

$$
\begin{gathered}
E F T\left(t_{a}\right) \leq \tau^{\prime}\left(t_{a}\right) \leq L F T\left(t_{a}\right) \\
\operatorname{Prob}\left\{\tau^{\prime}\left(t_{a}\right) \leq x\right\}=F_{t_{a}}(x)
\end{gathered}
$$

In the rest of this paper, we assume that $\operatorname{EFT}(t)<$ $\operatorname{LFT}(t)$, so as to exclude immediate and deterministic transitions. These could be encompassed in the theory that we present, but this would require a formulation based on the partitioned form of DBM regions [7] that would make the treatment much more tedious and long. As a side effect of the assumption, $\mathcal{C}$ becomes irrelevant to the purposes of the analysis [7], [9].

\section{Aggregation of stochastic State Classes}

\section{A. A simple but non-trivial case}

The net in Fig. 1 includes two GEN transitions $t_{0}$ and $t_{1}$ and a number of exponentially distributed (EXP) transitions that subtend a CTMC process subordinated to the periods in which $t_{0}$ and $t_{1}$ are persistent.

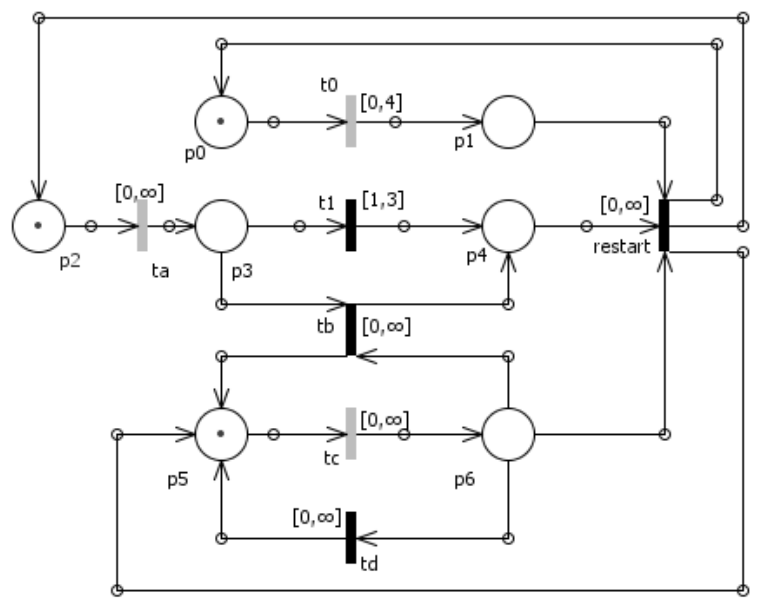

Fig. 1. A net with two GEN transitions, $t_{0}$ and $t_{1}$, and five EXP transitions, $t_{a}, t_{b}, t_{c}, t_{d}$ and restart. GEN transitions $t_{0}$ and $t_{1}$ are associated with firing density functions $f_{t_{0}}(x)=0.125 x$ over the support $[0,4]$ and $f_{t_{1}}(x)=$ $0.25 x$ over $[1,3]$, respectively. EXP transitions $t_{a}, t_{b}, t_{c}, t_{d}$ and restart have rates $\lambda_{a}=0.5, \lambda_{b}=1.2, \lambda_{c}=0.8, \lambda_{d}=0.85$ and $\lambda_{e}=0.9$, respectively.

Fig. 2 reports a fragment of the graph of non-deterministic state classes derived according to the theory of DBM domains [12], [10]. This identifies the reachable markings and the domain of times to fire under which markings can be reached through different execution sequences. For example, in $S_{0}$ we have three enabled transitions: $t_{0}$ with firing time in the interval $[0,4]$, and $t_{a}$ and $t_{c}$ with firing time in the interval $[0, \infty]$. If $t_{a}$ fires first, then the process arrives to the state class $S_{3}$ and $t_{1}$ gets enabled with a time to fire in $[1,3]$. If $t_{c}$ fires first in $S_{3}$ then the next state is $S_{9}$ in which we have four transitions with time to fire values constrained as reported in the figure. Starting from state class $S_{9}$, the firing of $t_{d}$ followed 
by the firing of $t_{c}$ constitutes a cycle in the graph. This depends on the fact that the boundaries of feasible times to fire returns to the same situation after the firing of $t_{d}$ and $t_{c}$.

The same firing sequence does not constitute a cycle in the stochastic state class graph. When constructing the stochastic state class graph, we consider not only the constraints of the time to fire values but their probability density function (pdf) as well. And for what concerns this pdf the firing of $t_{d}$ followed by the firing of $t_{c}$ brings the system into a new situation. In particular, the two exponential transitions $t_{c}$ and $t_{d}$ compose the CTMC that is subordinated to the period in which $t_{0}$ and $t_{1}$ are persistent after the entrance in class $S_{3}$. This subordinated CTMC can perform with positive probability any number of transitions before the firing of $t_{0}$ or $t_{1}$ occurs. Every firing of $t_{c}$ or $t_{d}$ changes the distribution of the time to fire values of $t_{0}$ and $t_{1}$ and hence gives rise to a new class in the graph of stochastic state classes. For this reason the stochastic state graph contains an infinite number of stochastic classes and the analysis of the model is not viable through the method of stochastic state classes [7], [9].

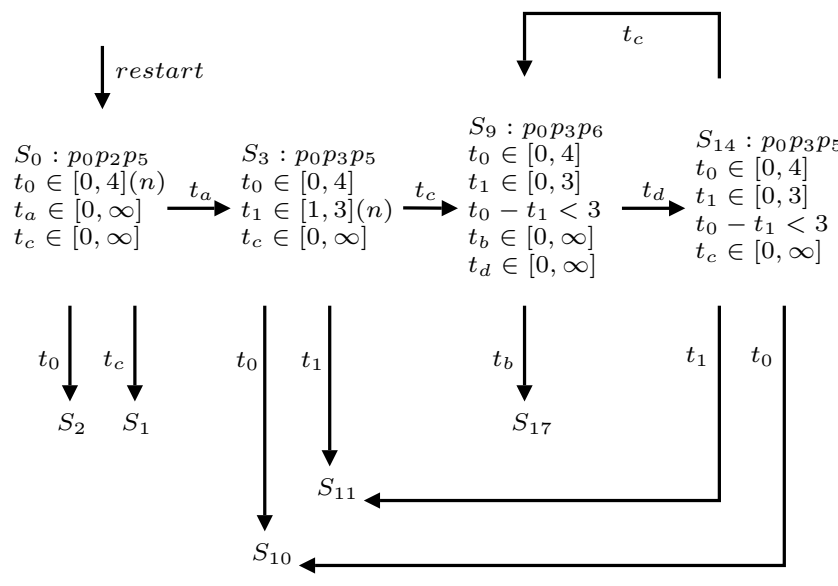

Fig. 2. A fragment of the graph of non-deterministic state classes of the net of Fig.1. Newly enabled transitions whose interval is not $[0, \infty]$ are marked by the symbol (n).

The net of Fig.1 cannot either be analysed by Markov Renewal Theory [2], [3] because its behaviour includes a period during which GEN transitions $t_{0}$ and $t_{1}$ are concurrently enabled with different times of enabling.

The net of Fig. 1 can be analysed through the method of supplementary variables [1] but only with very high computational costs. This is because the solution of the differential equations describing the evolution of the supplementary variables require discretisation and due to the presence of concurrently enabled general transitions, the discretized state space is huge. This could perhaps be afforded in the specific case of Fig.1 but would definitely be unpractical in any example where the number of reachable markings or the number of concurrently enabled GEN transitions increases.

Finally, the net cannot be analysed through synchronous sampling [4], as its GEN transitions are not deterministic.

Fig. 3 summarises the capabilities of the different analysis techniques. In the scheme, the horizontal direction accounts for increasing number of concurrently enabled GEN transitions (a single GEN under enabling restriction on the left, multiple concurrent GEN on the right), while the vertical direction accounts for increasing complexity of the firing time distributions (deterministic (DET) transitions on the bottom) and the complexity of the model increase. The schema omits to mention techniques that rely on approximation approaches. In this perspective, it is worth noting that the model could be analysed through the technique of [17] which used Bernstein polynomials to approximate state density functions over bounded supports. If GEN transitions are distributed over the infinite support $[0, \infty]$, approximation could be attained through continuous phase type distributions which reduce the model to Markovian behaviour [19].

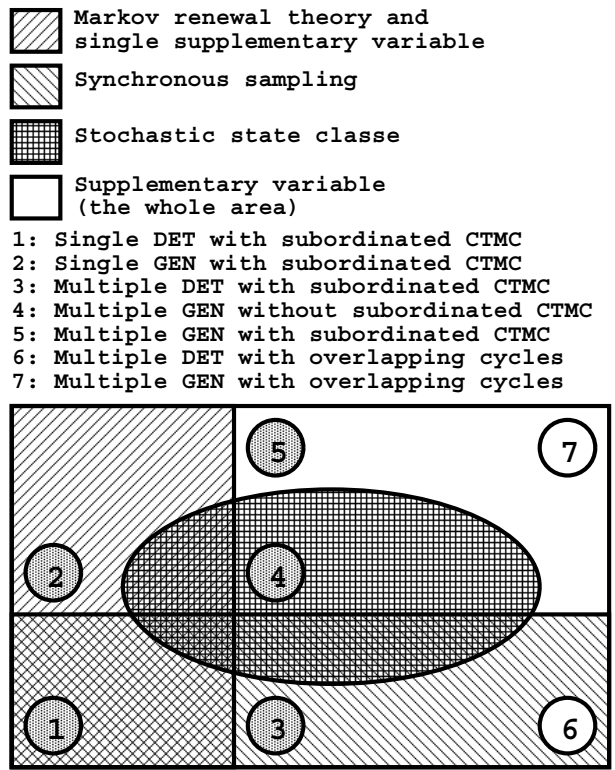

Fig. 3. Theoretical limits of applicability of different analysis techniques. The representation does not account for practical factors of complexity, which in particular affect the method of supplementary variables in the management of cases with multiple concurrently enabled transitions. The net in Fig.1 is an instance of class 5. Numbers with grey background colour are the cases that are encompassed in the theory of this paper.

\section{B. Aggregated stochastic state classes}

To overcome the limit, we generalise the concept of stochastic state class into a new abstraction which we call aggregated stochastic state class (aggregated class, for short). An aggregated class represents the behaviour of the net in a (maximal) period of activity during which the enabled GEN transitions are persistent. In so doing, the firing of any EXP transition that does not change the enabling status of any GEN transition is encapsulated into a single class.

In a conceptual perspective, class aggregation makes a half step back with respect to the refinement of the state space that underlies the theory of stochastic state classes [7], [9]: state classes distinguish states that share a common marking but have different support for enabled transitions, and stochastic state classes distinguish states where enabled transitions have the same support but a different distribution; vice versa, aggregated classes collect together stochastic state classes that 
may have different marking and different distribution of GEN transitions but are reached from each other through the firing of EXP transitions that do not change the enabling status of GEN transitions.

Formally, an aggregated class $\bar{\Sigma}$ is uniquely identified by an initial stochastic state class that determines the marking and the initial support and distribution of the vector of times to fire of enabled transitions:

Definition 3.1: an aggregated stochastic state class is a triple $\bar{\Sigma}=<M_{0}, D_{0}, f_{0}()>$, where $M_{0}$ is a marking, $D_{0}$ a domain for the vector of times to fire of transitions enabled in $M_{0}$, and $f_{0}()$ is a probability density function supported over $D_{0}$.

Domain $D_{0}$ is represented as the set of solutions of a set of linear inequalities in the form of a Difference Bounds Matrix (DBM). While a detailed discussion on DBM domains can be found in any of [7], [9], [12], for the purposes of the present treatment we recall that a DBM $D$ for a set of variables $\left\{\tau_{n}\right\}_{n=1}^{N}$ is expressed as:

$$
D=\left\{\begin{array}{l}
\tau_{i}-\tau_{j} \leq b_{i j} \\
\tau_{*}=0 \\
\forall i \neq j \in[1, N] \bigcup\{*\}
\end{array}\right.
$$

where $b_{i j} \in \mathbb{R} \bigcup\{\infty\}$ are values defining the borders of $D$, and they are said to be in normal form when $\forall i, j, h$ they satisfy the triangular inequality $b_{i j} \leq b_{i h}+b_{h j}$.

In the representation of $D_{0}$ and $f_{0}()$, EXP transitions can be omitted as their time to fire is always exponentially distributed over $[0, \infty]$.

The concept of aggregation takes meaning through the definition of the outgoing events and the succession relation between aggregated classes:

Definition 3.2: We say that $\left\langle M_{\text {out }}, t_{\text {out }}\right\rangle$ is an outgoing event from the aggregated class $\bar{\Sigma}=<M_{0}, D_{0}, f_{0}()>$, and we write $\bar{\Sigma}\left\langle M_{\text {out }}, t_{\text {out }}>\right.$, if there exists a firing sequence $\rho=$ $\rho_{\text {loc }} \rightarrow t_{\text {out }}$ where $\rho_{\text {loc }}$ is a possibly empty sequence of EXP transitions such that:

- $\rho$ is possible starting from the marking $M_{0}$;

- the firings of the sequence $\rho_{l o c}$ leads to marking $M_{o u t}$;

- the firings of the sequence $\rho_{l o c}$ does not disable or newly enable any GEN transition;

- $t_{\text {out }}$ is a GEN transition or it is an EXP transition whose firing disables or newly enables one or more GEN transitions.

According to the definition, the firings of $t_{\text {out }}$ from different markings are regarded as distinct events; whereas, firings of $t_{\text {out }}$ after different prefixes $\rho_{\text {loc }}$ that reach the same local marking $M_{\text {out }}$ are regarded as instances of the same outgoing event.

Definition 3.3: $\bar{\Sigma}^{\prime}=<M^{\prime}, D^{\prime}, f^{\prime}()>$ is the successor of $\bar{\Sigma}=<M_{0}, D_{0}, f_{0}()>$ through $<M_{\text {out }}, t_{\text {out }}>$, and we write $\bar{\Sigma}<\stackrel{M_{\text {out }}, t_{\text {out }}>}{\longrightarrow} \bar{\Sigma}^{\prime}$, if the following property holds: if the net is initially in the marking $M_{0}$ and the vector of times to fire of enabled GEN transitions is distributed over $D_{0}$ according to $f_{0}()$, then $\stackrel{<M_{\text {out }}, t_{\text {out }}>}{\longrightarrow}$ is an outgoing event of $\bar{\Sigma}$ and it leads to marking $M^{\prime}$ with the vector of times to fire of enabled GEN transitions distributed over $D^{\prime}$ according to $f^{\prime}()$.

\section{Calculus of Aggregated Stochastic Classes}

We develop here the calculus of the succession relation among aggregated classes.

\section{A. Evolution of the subordinated CTMC}

According to Def.3.2, the sojourn within an aggregated class encompasses the evolution of a subordinated CTMC whose states correspond to the markings that can be reached from the initial marking of the class, $M_{0}$, through the firing of one or more EXP transitions that do not change the enabling status of the GEN transitions.

The behaviour of the subordinated CTMC will be described by its infinitesimal generator denoted by $Q$. The off-diagonal entries of $Q$ are given by the rates of the EXP transitions that do not change the enabling status of the GEN transitions. The diagonal entries instead contain the sum of the rates of all the EXP transitions that are enabled in the corresponding marking. As a consequence, if there are one or more states in the subordinated CTMC in which there are one or more exponential transitions that change the enabling situation of the GEN transitions, then $Q$ is defective, i.e., not all row sums of $Q$ are 0 . As a result, the row sums of $\exp (Q x)$ is not 1 and this describes appropriately the fact that the subordinated CTMC can be left due to the firing of an exponential transition whose firing changes the enabling situation of the GEN transitions. Having so defined $Q$, the probability that the subordinated CTMC is in marking $M_{\text {out }}$ at time $x$ is given by

$$
P_{M_{\text {out }}}(x)=[\exp (Q x)]_{0, \text { out }}
$$

where $[\exp (Q x)]_{0, \text { out }}$ is that entry of the matrix $\exp (Q x)$ whose row index is the one corresponding to marking $M_{0}$ and whose column index is the one corresponding to marking $M_{\text {out }}$. We will denote by $M(x)$ the random variable that gives the marking of the subordinated CTMC at time $x$.

\section{B. Feasibility and probability of outgoing events}

EXP transitions in the subordinated CTMC are not subject to any bound on the time of firing. According to this, for any EXP transition $t_{\text {out }}$, the pair $\left\langle M_{\text {out }}, t_{\text {out }}\right\rangle$ is a feasible outgoing event iff $M_{\text {out }}$ is present in in the subordinated CTMC, $t_{\text {out }}$ is enabled in $M_{\text {out }}$, and the firing of $t_{\text {out }}$ changes the enabling status of the GEN transitions.

On the contrary, GEN transitions are in general subject to constraints on their firing time. Accordingly, if $t_{\text {out }}$ is a GEN transition, then $\left\langle M_{\text {out }}, t_{\text {out }}\right\rangle$ is an outgoing event iff $M_{\text {out }}$ is present in the subordinated CTMC and the domain $D_{0}$ of the aggregated class accepts solutions where the time to fire of $t_{\text {out }}$ is not higher than the firing time of any other enabled GEN transition. As shown in [7], [9], this occurs iff $b_{i, \text { out }} \geq 0$ for every enabled GEN $t_{i}$, where $b_{. .}$are the coefficients of the normal form of $D_{0}$.

In the following, we derive the probability of the outgoing events. We denote by $t_{i}, 0 \leq i \leq N-1$, the enabled GEN transitions and assume, without loss of generality, that if the outgoing event is due to the firing of a GEN transition then this transition is $t_{0}$, i.e., $t_{\text {out }}=t_{0}$. 
The probability $P_{e v}$ that the aggregated class is abandoned through the event $e v=\left\langle M_{\text {out }}, t_{\text {out }}\right\rangle$ can be expressed as:

$$
P_{e v}=\int_{E F T_{e v}}^{L F T_{e v}} f_{\tau_{e v}}(x) d x
$$

where $f_{e v}()$ is the density function of the time of occurrence of the the event $e v$, while $E F T_{e v}$ and $L F T_{e v}$ are the earliest and latest extrema of its support. On the one hand, $L F T_{e v}$ is the minimum among the latest firing times of the enabled GEN transitions and can thus be expressed as

$$
L F T_{e v}=\min _{t_{i} \in G E N}\left\{b_{i *}\right\}
$$

where $b_{\text {.. }}$ are the coefficients of the normal form of $D_{0}$. On the other hand, the lower extremum $E F T_{e v}$ has a different form for EXP and GEN transitions: while EXP transitions have no lower bound on the firing time, the firing time of a GEN transition may be lower bounded:

$$
E F T_{\text {ev }}= \begin{cases}-b_{*, \text { out }} & \text { if } t_{\text {out }} \in G E N \\ 0 & \text { if } t_{\text {out }} \in E X P\end{cases}
$$

The density function $f_{\tau_{e v}}(x)$ is derived in a different manner depending on whether $t_{\text {out }}$ is a GEN or an EXP transition.

On the one hand, in the case when $t_{\text {out }}$ is a GEN transition, $\left\langle M_{\text {out }}, t_{\text {out }}\right\rangle$ is the outgoing event and occurs at time $x$ iff the time to fire of $t_{\text {out }}$ is $x$ and the time to fire of any other GEN transition is not lower than $x$ and the marking at time $x$ is $M_{\text {out }}$. According to this, since the firing times of GEN transitions are independent from the evolution of the subordinated CTMC:

$$
\begin{aligned}
& f_{\tau_{\text {ev }}}(x)=\lim _{d x \rightarrow 0} \frac{\operatorname{Prob}\left\{\tau_{\text {ev }} \in[x, x+d x]\right\}}{d x}= \\
& =\lim _{d x \rightarrow 0} \frac{1}{d x} \operatorname{Prob}\left\{\left(M(x)=M_{\text {out }}\right) \wedge\left(\tau_{\text {out }} \in[x, x+d x]\right) \wedge\right. \\
& \left.\left(\tau_{i} \geq x+d x, 1 \leq i \leq N-1\right)\right\}= \\
& =P_{M_{\text {out }}}(x) \cdot \int_{D_{x}^{\text {out }}} f_{\left\langle\tau_{\text {out }}, \underline{\tau}\right\rangle}(x, \underline{y}) d \underline{y}
\end{aligned}
$$

where $\tau$ is the vector of times to fire of all enabled GEN transitions other than $t_{\text {out }}$, and $D_{x}^{\text {out }}$ is a domain for $\underline{\tau}$ derived from $D_{0}$ by imposing that the time to fire of $t_{\text {out }}$ is equal to $x$ and that all GEN transitions other than $t_{\text {out }}$ have a time to fire higher than $x$ :

$$
D_{x}^{\text {out }}=\left\{\underline{y} \mid\left(\langle x, \underline{y}\rangle \in D_{0}\right) \wedge\left(x \leq y_{i}, 1 \leq i \leq N-1\right)\right\}
$$

On the other hand, in the case when $t_{\text {out }}$ is an EXP transition with rate $\lambda_{\text {out }}$, the event $\left\langle M_{\text {out }}, t_{\text {out }}\right\rangle$ occurs at time $x$ iff the time to fire of all GEN transitions are not lower than $x$, the marking at time $x$ is $M_{\text {out }}$ and $t_{\text {out }}$ fires at time $x$. According to this:

$$
\begin{aligned}
f_{\tau_{\text {ev }}}(x) & =\lim _{d x \rightarrow 0} \frac{1}{d x} \operatorname{Prob}\left\{\left(M(x)=M_{\text {out }}\right) \wedge\right. \\
\wedge\left(\tau_{\text {out }}\right. & \left.\in[x, x+d x]) \wedge\left(\tau_{i} \geq x+d x, 0 \leq i \leq N-1\right)\right\}= \\
= & P_{M_{\text {out }}}(x) \cdot \lambda_{\text {out }} \cdot \int_{D_{x}} f_{\langle\underline{\tau}\rangle}(\underline{y}) d \underline{y}
\end{aligned}
$$

where $\langle\underline{\tau}\rangle$ is the vector of times to fire of the enabled GEN transitions, and $D_{x}$ restricts $D_{0}$ with the assumption that all enabled GEN transitions have a time to fire not lower than $x$ :

$$
D_{x}=\left\{\langle\underline{y}\rangle \in D_{0} \mid x \leq y_{i}, 0 \leq i \leq N-1\right\} \text {. }
$$

C. State density function in the aggregated class reached through the firing of a GEN transition

In this section we derive the state density function for an aggregated class reached through the firing of a GEN transition. We assume that the firing occurs in the marking $M_{\text {out }}$ and the transition that fires is $t_{0}=t_{\text {out }}$, i.e., the outgoing event is $\left\langle M_{\text {out }}, t_{\text {out }}\right\rangle$. The GEN transitions that are enabled in $M_{\text {out }}$ are denoted by $t_{\text {out }}, t_{1}, \ldots t_{N-1}$. The domain and the pdf of the times to fire in the originating aggregated class is denoted by $D$ and $f_{\underline{\tau}}()$, respectively. The vector $\underline{\tau}=\left\langle\tau_{\text {out }}, \tau_{1}, \ldots \tau_{N-1}\right\rangle$ is composed of the random variables describing the times to fire of the GEN transitions.

The assumption that $\tau_{\text {out }}$ is not higher than the time to fire of any other enabled GEN transition conditions $\underline{\tau}$ and yields a new vector of random variables $\underline{\tau}^{a}$ :

$$
\underline{\tau}^{a}=\underline{\tau} \mid\left(\tau_{\text {out }} \leq \tau_{i}, 1 \leq i \leq N-1\right)
$$

whose support $D^{a}$ and density function $f_{\tau^{a}}()$ are given as

$$
\begin{aligned}
& D^{a}=\left\{\begin{array}{l}
D \\
\tau_{\text {out }} \leq \tau_{i} \quad 1 \leq i \leq N-1
\end{array}\right. \\
& f_{\underline{\tau}^{a}}\left(x_{\text {out }}, x_{1}, \ldots x_{N-1}\right)=\frac{f_{\underline{\tau}}\left(x_{\text {out }}, x_{1}, \ldots x_{N-1}\right)}{P_{\text {out }- \text { first }}}
\end{aligned}
$$

where

$$
P_{\text {out-first }}=\int_{D^{a}} f_{\tau}\left(y_{\text {out }}, y_{1}, \ldots y_{N-1}\right) d y_{\text {out }} d y_{1} \ldots d y_{N-1} .
$$

The assumption that the firing of $t_{\text {out }}$ occurs while the marking is $M_{\text {out }}$ conditions $\underline{\tau}^{a}$, yielding a new vector of random variables $\underline{\tau}^{b}$ :

$$
\underline{\tau}^{b}=\underline{\tau}^{a} \mid M\left(\tau_{\text {out }}\right)=M_{\text {out }}
$$

According to (2) and (3), the domain $D^{b}$ of $\tau^{b}$ is equal to $D^{a}$. However, since the time of the firing has an impact on the probability that the underlying CTMC is in the marking $M_{\text {out }}$, the assumption $M\left(\tau_{\text {out }}\right)=M_{\text {out }}$ changes the distribution of $\underline{\tau}^{b}$ :

$$
\begin{aligned}
& f_{\underline{\tau}^{b}}\left(x_{\text {out }}, x_{1}, \ldots x_{N-1}\right)= \\
& \left.\int_{D^{a}} f_{\underline{\tau}^{a}}\left(y_{\text {out }}, y_{1}, \ldots y_{N-1}\right) P_{M_{\text {out }}}\left(y_{\text {out }}\right) d y_{\text {out }}, d y_{1}, \ldots d y_{N-1}\right) \\
& .
\end{aligned}
$$

When $t_{\text {out }}$ fires, the time to fire of each transition other than $t_{\text {out }}$ is reduced by the value of $\tau_{\text {out }}^{a}$ and $\tau_{\text {out }}^{a}$ itself is eliminated. This yields once again a new vector of random variables $\underline{\tau}^{c}$ which is of length $N-1$ :

$$
\underline{\tau}^{c}=\left\langle\tau_{1}^{b}-\tau_{\text {out }}^{b}, \tau_{2}^{b}-\tau_{\text {out }}^{b}, \ldots \tau_{N-1}^{b}-\tau_{\text {out }}^{b}\right\rangle
$$

The domain $D^{c}$ is derived by shifting $D^{b}$ by $\tau_{\text {out }}^{b}$ and then by computing the projection that eliminates $\tau_{\text {out }}^{b}$ itself. The density function $f_{\tau^{c}}()$ is derived by shifting all the times to fire by $\tau_{\text {out }}^{b}$ and by eliminating $\tau_{\text {out }}^{b}$ itself through a projection:

$$
\begin{aligned}
& f_{\underline{\tau}^{c}}\left(x_{1}, \ldots, x_{N-1}\right)=\int_{E F T_{\text {out }}\left(x_{1}, \ldots x_{N-1}\right)}^{L F T_{\text {out }}\left(x_{1}, \ldots x_{N-1}\right)} \\
& f_{{\underline{\tau^{b}}}^{b}}\left(x_{\text {out }}, x_{1}+x_{\text {out }}, \ldots, x_{N-1}+x_{\text {out }}\right) d x_{\text {out }}
\end{aligned}
$$

where $\operatorname{EFT}_{\text {out }}\left(x_{1}, \ldots x_{N-1}\right)$ and $\operatorname{LFT}_{\text {out }}\left(x_{1}, \ldots x_{N-1}\right)$ are the minimum and maximum value of $x_{\text {out }}$ such that $\left\langle x_{\text {out }}, x_{1}+\right.$ 
$\left.x_{\text {out }}, \ldots, x_{N-1}+x_{\text {out }}\right\rangle$ is in the support of $\tau^{b}$. For details on the derivation of $E F T_{o u t}\left(x_{1}, \ldots x_{N-1}\right)$ and $L F T_{\text {out }}\left(x_{1}, \ldots x_{N-1}\right)$, see [7], [9].

GEN transitions that get disabled at the firing of $t_{0}$ are eliminated through subsequent projection operations. For instance, if $t_{N-1}$ is not persistent, its elimination yields a new vector of random variables $\underline{\tau}^{d}=\left\langle\tau_{1}^{c}, \ldots \tau_{N-2}^{c}\right\rangle$, supported within the projection of $D^{c}$ that eliminates $\tau_{N-1}^{c}$ and distributed according to the density function:

$$
\begin{aligned}
& f_{\tau^{d}}\left(x_{1}, \ldots x_{N-2}\right)= \\
& \int_{E F T_{N-1}\left(x_{1}, \ldots x_{N-2}\right)}^{L F T_{N-1}\left(x_{1}, \ldots x_{N-2}\right)} f_{\underline{\tau}^{c}}\left(x_{1}, \ldots, x_{N-2}, y_{N-1}\right) d y_{N-1}
\end{aligned}
$$

where $\left[E F T_{N-1}\left(x_{1}, \ldots x_{N-1}\right), L F T_{N-1}\left(x_{1}, \ldots x_{N-1}\right)\right]$ is the support of $\tau_{N-1}^{c}$ under the constraint that $\tau_{1}^{c}=$ $x_{1}, \tau_{2}^{c}=x_{2}, \ldots, \tau_{N-2}^{c}=x_{N-2}$. As before, for details on $E F T_{N-1}\left(x_{1}, \ldots x_{N-1}\right)$ and $L F T_{N-1}\left(x_{1}, \ldots x_{N-1}\right)$, see [7], [9].

In the final step of the derivation, the vector of times to fire is extended with the times to fire of the GEN transitions that are newly enabled after the firing of $t_{0}$. According to the semantics of the model, newly enabled transitions sample their times to fire independently. Consequently, the resulting function is the product of the multivariate density function of the persistent GEN transitions by the density function of each newly enabled GEN transition.

\section{State density function in the aggregated class reached through the firing of an EXP transition}

The derivation is slightly changed in the case that the transition from one aggregated class to another is due to the firing of an EXP transition.

In this case, let $\underline{t}=\left\langle t_{1}, \ldots t_{N-1}\right\rangle$ be the vector of GEN transitions enabled in the originating class, and let $D$ and $f_{\underline{\tau}}()$ be the support and the distribution of their times to fire, respectively. The vector composed of the random variables describing the times to fire is denoted by $\underline{\tau}=\left\langle\tau_{1}, \ldots \tau_{N-1}\right\rangle$.

The assumption that the subordinated CTMC is abandoned through the firing of an EXP transition $t_{\text {out }}$ conditions the random variables $\underline{\tau}=\left\langle\tau_{1}, \ldots \tau_{N-1}\right\rangle$. This fact is taken into account by defining a new vector of random variables as

$$
\begin{aligned}
& \frac{\tau}{}^{a}=\left\langle\tau_{\text {out }}^{a}, \underline{\tau}\right\rangle \mid \\
& \left(\tau_{\text {out }} \leq \tau_{n}, 1 \leq n \leq N-1\right) \wedge\left(M\left(\tau_{\text {out }}\right)=M_{\text {out }}\right)
\end{aligned}
$$

with support $D^{a}$ and density function $f_{\underline{\tau}}$ given as

$$
\begin{aligned}
& D^{a}=\left\{\begin{array}{l}
\left\langle\tau_{\text {out }}^{a}, \underline{\tau}\right\rangle \in[0, \infty] \times D \\
\tau_{\text {out }}^{a} \leq \tau_{n}, 1 \leq n \leq N-1
\end{array}\right. \\
& f_{\underline{\tau}^{a}}\left(x_{\text {out }}, x_{1}, \ldots x_{N-1}\right)= \\
& =\frac{f_{\underline{\tau}}\left(x_{1}, \ldots x_{N-1}\right) P_{M_{\text {out }}}\left(x_{\text {out }}\right) \lambda_{\text {out }}}{\int_{D^{a}} f_{\underline{\tau}}\left(y_{1}, \ldots y_{N-1}\right) P_{M_{\text {out }}}\left(y_{\text {out }}\right) \lambda_{\text {out }} d y_{\text {out }} d y_{1} \ldots d y_{N-1}} .
\end{aligned}
$$

When $t_{\text {out }}$ fires, the time to fire of each transition other than $t_{\text {out }}$ is reduced by the value of $\tau_{\text {out }}^{a}$ and $\tau_{\text {out }}^{a}$ itself is eliminated. This yields a new vector of random variables $\tau^{b}$ of length $N-1$

$$
\tau^{b}=\left\langle\tau_{1}^{a}-\tau_{\text {out }}^{a}, \tau_{2}^{a}-\tau_{\text {out }}^{a}, \ldots \tau_{N-1}^{a}-\tau_{\text {out }}^{a},\right\rangle
$$

whose domain $D^{b}$ is derived by shifting $D^{a}$ by $\tau_{\text {out }}^{a}$ and then by computing the projection that eliminates $\tau_{\text {out }}^{a}$ itself. The density function $f_{\tau^{b}}()$ is derived by shifting all the times to fire by $\tau_{\text {out }}^{a}$ and by eliminating $\tau_{\text {out }}^{a}$ itself through a projection:

$$
\begin{aligned}
f_{\underline{\tau}^{b}}\left(x_{1}, \ldots x_{N-1}\right) & =\int_{E F T_{\text {out }}\left(x_{1}, \ldots x_{N-1}\right)}^{L F T_{\text {out }}\left(x_{1}, \ldots x_{N-1}\right)} \\
& f_{\underline{\tau}^{a}}\left(y_{\text {out }}, y_{1}+y_{\text {out }}, \ldots, y_{N-1}+y_{\text {out }}\right) d y_{\text {out }}
\end{aligned}
$$

where $\left[E F T_{\text {out }}\left(x_{1}, \ldots x_{N-1}\right), L F T_{\text {out }}\left(x_{1}, \ldots x_{N-1}\right)\right]$ are the minimum and maximum value for $x_{\text {out }}$ such that $\left\langle x_{\text {out }}, x_{1}+\right.$ $\left.x_{\text {out }}, \ldots, x_{N-1}+x_{\text {out }}\right\rangle$ is in the support of $\underline{\tau}^{a}$. Once again, for details on $E F T_{\text {out }}\left(x_{1}, \ldots x_{N-1}\right)$ and $L F T_{\text {out }}\left(x_{1}, \ldots x_{N-1}\right)$, see [7], [9].

The derivation of the final form of the support and distribution of the vector of GEN transitions enabled in the newly reached aggregated class is completed through the elimination of disabled transitions and the addition of newly enabled transitions as described in Sect:IV-C.

\section{E. Graph enumeration and termination}

The results of Sections IV-A through IV-D can be embedded in a conventional enumeration semi-algorithm that builds an aggregated stochastic class graph (aggregated graph, for short) representing the succession relation among aggregated classes. Fig.4 reports the aggregated graph for the net in Fig.1.

A core step of the enumeration is the symbolic derivation of the pdf of the vector of times to fire. This requires the symbolic solution of integrals that include the exponential of the matrix $Q, \exp (Q x)$, multiplied by expolynomial functions. For small or structured subordinated CTMCs, $\exp (Q x)$ can be expressed in closed form and hence the integrals can be obtained in a fully symbolic manner. When $\exp (Q x)$ is not available in closed form, a viable solution, as proposed in [17], consists in calculating the integrals numerically by uniformisation of the subordinated CTMC [20] for a set of sample points and then using the samples to construct a Bernstein polynomial to approximate the pdf of the vector of times to fire. This approach, which results in an approximation, allows for evaluating nets with large subordinated CTMCs.

The aggregated graph can be seen as the stochastic expansion of the graph of non-deterministic state classes. In fact, by construction, each aggregated class $\bar{\Sigma}=\langle M, D, f\rangle$ is associated with a unique state class $S=\langle M, D\rangle$ which we say to be its projection. In general, a state class can be the projection of several aggregated classes that share the same marking and firing domain but have a different probability distribution.

A state class is said to be a regeneration class if all its enabled GEN transitions are newly enabled [7], [9]. Intuitively, a regeneration class is a point where the underlying process of the net looses memory of its previous history. The entry into a regeneration class corresponds to the concept of regeneration point of [2]. A regeneration class is always the projection of one and only one aggregated class: on the one hand, in an aggregated class that projects onto a regeneration class, all enabled GEN transitions are newly enabled, and their density is thus uniquely determined as the product of the probability 


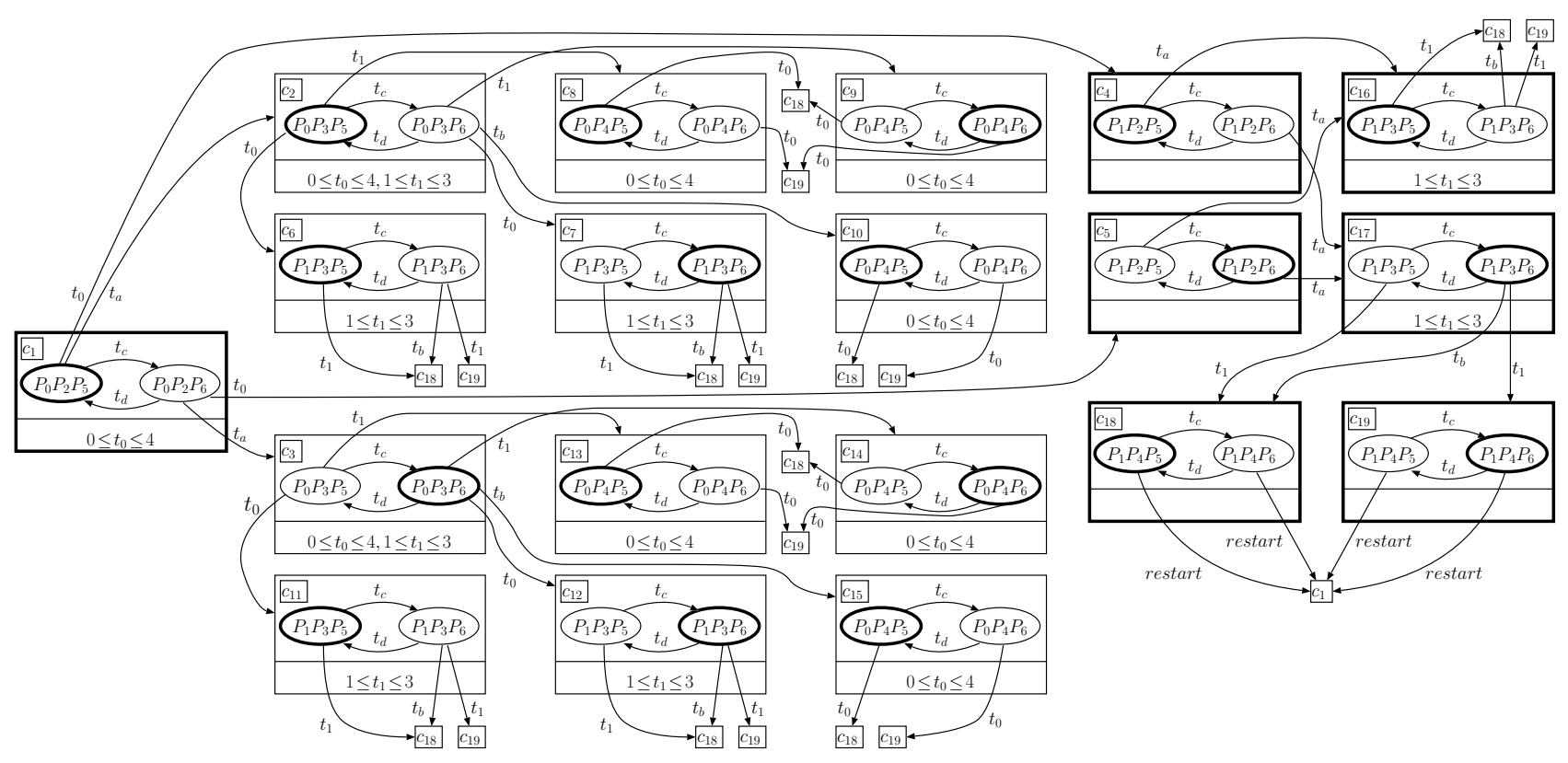

Fig. 4. The aggregated class graph for the net of Fig.1. In the visual representation, each aggregated class is represented by a rectangle depicted with: the name of the class (e.g., $c_{3}$ ); the subordinated CTMC whose states are labelled with the corresponding marking and whose transitions are labelled with the corresponding EXP transition; and the firing domain of the enabled GEN transitions. Classes depicted with thicker borders are regenerative, i.e., either there is no GEN transition present or all GEN transitions are newly enabled. The initial state of each subordinated CTMC is signed by thicker borders. Transitions among the classes are labelled by the corresponding EXP or GEN transition. In order to have a clearer figure, the end point of several transitions are indicated only by the label of the class where they should arrive.

density functions of enabled GEN transitions; on the other hand, since all its enabled GEN transitions are newly enabled, a regeneration class $S^{*}$ cannot be reached through firings that do not change the enabling status of GEN transitions.

The enumeration of the aggregated graph terminates under a condition that extends the limits of applicability of the theory of stochastic state classes:

Theorem 4.1: If the graph of non-deterministic state classes is finite and every cycle that it includes either maintains the enabling status of GEN transitions or visits at least on regeneration class, then also the aggregated graph is finite.

Proof: Assuming by absurd that the aggregated graph is unbounded, it must include an infinite path $\bar{\rho}$ that never visits twice the same aggregated class. We call $\rho$ the "projection" of $\bar{\rho}$ on the class graph. Since the class graph is finite, $\rho$ must be a cycle.

Along $\rho$, the enabling status of GEN transitions cannot be maintained, otherwise $\bar{\rho}$ could not visit more than one single aggregated class. According to the hypotheses of the Theorem, since $\rho$ is a cycle that changes the enabling status of GEN transitions, it must visit at least one regeneration class $S^{*}$. And since $S^{*}$ is a regeneration class, there exists uniquely one aggregated class $\bar{\Sigma}^{*}$ that projects onto $S^{*}$.

Since $\rho$ is a cycle, $\bar{\rho}$ must visit an infinite number of times an aggregated class that projects onto $S^{*}$. However, since the aggregated class that projects on $S^{*}$ exists uniquely, the aggregated class $\bar{\Sigma}^{*}$ is visited infinitely often along $\bar{\rho}$, which is not possible as all aggregated classes visited along $\bar{\rho}$ are different.

Remark 4.1: The conditions for the application of the Theorem can be verified on the class graph before its stochastic

\section{expansion.}

Remark 4.2: Referring to the classification of Fig.3, Theorem 4.1 extends the limits of applicability of the theory of stochastic state classes by including the classes of models $1,2,3,5$ which include a CTMC underlying the period of persistence of one or more GEN transitions. In this way, the class of models that are amenable to steady state analysis through aggregated classes becomes a proper extension of the class of models that can be managed through Markov Renewal Theory [2], [3], through the method of Supplementary (Single) Variable [1], [6] or through the theory of stochastic state classes [8], [9], [7].

\section{F. Analysis of the aggregated stochastic graph}

Based on the aggregated stochastic graph, it is possible to compute the steady state probabilities of the markings. This requires the following steps.

The aggregated graph provides the embedded DTMC of the aggregated stochastic state classes. From this not necessarily ergodic DTMC we can obtain the embedded steady state probabilities of the classes which we will denote by $\pi_{i}^{(e)}$. Considering a given class, applying the results of Section IV-B the pdf of the delay associated with a given outgoing event can be obtained. Having derived this pdf for every possible outgoing event, it is straightforward to compute the mean sojourn time of the classes. Let us denote the mean sojourn time of class $i$ by $S_{i}$. The steady state probability of a given class, denoted by $\pi_{i}$, can then be obtained by considering the 
embedded steady state probabilities and the sojourn times as

$$
\pi_{i}=\frac{S_{i} \pi_{i}^{(e)}}{\sum_{j} S_{j} \pi_{j}^{(e)}}
$$

The next step is to distribute the steady state probability of the classes among the markings that are incorporated in the subordinated CTMCs. In order to deal with this problem, let us concentrate on class $i$ and denote its joint pdf of the time to fire values by $f_{i}(\underline{x})$, the support of $f_{i}(\underline{x})$ by $D_{i}$, the infinitesimal generator of its subordinated CTMC by $Q_{i}$ and the number of subordinated markings by $N_{i}$. Further, let us denote by $H_{i}(x)$ the probability that all the GEN transitions have firing time larger than $x$. For classes without enablad GEN transitions $H_{i}(x)=1$. This quantity can be obtained as

$$
H_{i}(x)=\int_{\underline{x} \in D_{i}, \forall j: x_{j}>x} f_{i}(\underline{x}) d \underline{x} .
$$

We calculate for each subordinated marking $j, 1 \leq j \leq N_{i}$, the quantity

$$
k_{i, j}=\int_{0}^{\infty} H_{i}(x)\left[\exp \left(Q_{i} x\right)\right]_{1, j} d x .
$$

which determine the proportions according to which the steady state probability of class $i$ has to be distributed among its subordinated markings. Consequently, the steady state probability of marking $j$ inside class $i$, denoted by $\pi_{i, j}$, is obtained by

$$
\pi_{i, j}=\frac{k_{i, j}}{\sum_{j=1}^{N_{i}} k_{i, j}} \pi_{i}
$$

Since the same marking can be present in more than one class, the final step is to sum up for all markings those $\pi_{i, j} \mathrm{~s}$ that correspond to the marking.

\section{ILLUSTRATIVE EXAMPLE}

To illustrate the proposed technique, we report here part of the calculus for the Petri net depicted in Fig.1. While this example represents a class of non-trivial cases, its subordinated CTMCs are simple and have such transient probabilities that can be written in closed form. This permits the explicit representation of the concepts of the analysis technique.

The aggregated class graph of the model is given in Fig.1. The initial marking is $P_{0} P_{2} P_{5}$ and we start the construction of the aggregated graph from class 1 corresponding to this marking. In class 1 we have one GEN transition and it is newly enabled. Consequently, the pdf of the time to fire is given simply by the pdf of transition $t_{0}$, i.e., $f_{c_{1}}\left(x_{0}\right)=0.125 x_{0}$ over the support $[0,4]$. The subordinated CTMC has two states corresponding to markings $P_{0} P_{2} P_{5}$ and $P_{0} P_{4} P_{6}$, it is started in its first state and its infinitesimal generator is

$$
Q_{c_{1}}=\left|\begin{array}{cc}
-\lambda_{c}-\lambda_{a} & \lambda_{c} \\
\lambda_{d} & -\lambda_{d}-\lambda_{a}
\end{array}\right|=\left|\begin{array}{cc}
-1.3 & 0.8 \\
0.85 & -1.35
\end{array}\right|
$$

indicating that firings of transitions $t_{c}$ and $t_{d}$ do not change the enabling situation of the GEN transitions and hence do not lead to a transition from class 1 to another class while the firing of $t_{a}$ does. Note that the subordinated CTMC is defective (its row sums are not 0 ) and this implies that the entries of $\exp \left(Q_{c_{1}} t\right)$ tend to 0 as $t$ tend to infinity.
First we calculate the probability of the outgoing events of class 1 according to Section IV-B. There are four different ways of leaving class 1: firing of EXP transition $t_{a}$ in marking $P_{0} P_{2} P_{5}$ or $P_{0} P_{2} P_{6}$ (leading to class 2 and 3 , respectively), firing of GEN transition $t_{0}$ in marking $P_{0} P_{2} P_{5}$ or $P_{0} P_{2} P_{6}$ (leading to class 4 and 5 , respectively). Let us consider first the firing of $t_{0}$ in marking $P_{0} P_{2} P_{5}$. This event happens if the firing of $t_{0}$ occurs while the subordinated CTMC is in its first state. We have

$$
p_{c_{1}, c_{4}}=\int_{x_{0}=0}^{4}\left[\exp \left(Q_{c_{1}} x_{0}\right)\right]_{1,1} f_{c_{1}}\left(x_{0}\right) d x_{0} .
$$

The probability $p_{c_{1}, c_{5}}$ can be obtained similarly as

$$
p_{c_{1}, c_{5}}=\int_{x_{0}=0}^{4}\left[\exp \left(Q_{c_{1}} x_{0}\right)\right]_{1,2} f_{c_{1}}\left(x_{0}\right) d x_{0} .
$$

The probability that class 1 is left due to the firing of $t_{a}$ occuring in marking $P_{0} P_{2} P_{5}$ is given by

$$
p_{c_{1}, c_{2}}=\int_{x_{a}=0}^{4} \int_{x_{0}=x_{a}}^{4}\left[\exp \left(Q_{c_{1}} x_{a}\right)\right]_{1,1} \lambda_{a} f_{c_{1}}\left(x_{0}\right) d x_{0} d x_{a}
$$

where the term $\left[\exp \left(Q_{c_{1}} x_{a}\right)\right]_{1,1} \lambda_{a}$ is the firing intensity of transition $t_{a}$ at time $x_{a}$ and the integrals reflect the fact that GEN transition $t_{0}$ does not fire before the firing of $t_{a}$. Similarly

$$
p_{c_{1}, c_{3}}=\int_{x_{a}=0}^{4} \int_{x_{0}=x_{a}}^{4}\left[\exp \left(Q_{c_{1}} x_{a}\right)\right]_{1,2} \lambda_{a} f_{c_{1}}\left(x_{0}\right) d x_{0} d x_{a} .
$$

The next step is to characterise the classes reached from class 1 . We start from class 2 . In this class there are two GEN transitions: $t_{0}$ which is not newly enabled and $t_{1}$ which is newly enabled. The joint pdf of the times to fire of these two transitions is obtained as $f_{c_{2}}\left(x_{0}, x_{1}\right)=$

$$
\frac{1}{p_{c_{1}, c_{2}}} f_{t_{1}}\left(x_{1}\right) \int_{x_{a}=0}^{4-x_{0}}\left[\exp \left(Q_{c_{1}} x_{a}\right)\right]_{1,1} \lambda_{a} f_{c_{1}}\left(x_{0}+x_{a}\right) d x_{a} \text {. }
$$

with support $0 \leq x_{0} \leq 4,1 \leq x_{1} \leq 3$. The interpretation of the above expression is as follows. The division by $p_{c_{1}, c_{2}}$ guarantees a normalised pdf. The pdf of transition $t_{1}, f_{t_{1}}\left(x_{1}\right)$, is present because this transition is newly enabled in class 2 . The integral takes into account the fact that the pdf of the time to fire of transition $t_{0}$, which was given by $f_{c_{1}}\left(x_{0}\right)$ in class 1 , is shifted by a random quantity $x_{a}$ whose pdf is proportional to $\left[\exp \left(Q_{c_{1}} x_{a}\right)\right]_{1,1} \lambda_{a}$. The limit of the integral is due to the fact that in order to have $x_{0}$ as time to fire of transition $t_{0}$ in class 2 , the firing time of transition $t_{a}$ in class 1 must be smaller than $4-x_{0}$.

The infinitesimal generator of the subordinated CTMC of class 2 is

$$
Q_{c_{2}}=\left|\begin{array}{cc}
-\lambda_{c} & \lambda_{c} \\
\lambda_{d} & -\lambda_{d}-\lambda_{b}
\end{array}\right|=\left|\begin{array}{cc}
-0.8 & 0.8 \\
0.85 & -1.65
\end{array}\right|
$$

which is once again a defective CTMC indicating that firings of transitions $t_{c}$ and $t_{d}$ do not cause a transition from class 2 to another class while firing of $t_{b}$ does. The initial state of the subordinated CTMC is state 1 .

There are five different ways of leaving class 2 . The two GEN transitions, $t_{0}$ and $t_{1}$, can fire both in $P_{0} P_{3} P_{5}$ and 
$P_{0} P_{3} P_{6}$ while the EXP transition $t_{b}$ can fire only in $P_{0} P_{3} P_{6}$. The resulting classes are $c_{i}, 6 \leq i \leq 10$ (see Fig.1). The probability that $t_{0}$ fires in $P_{0} P_{3} P_{5}$ is obtained as $p_{c_{2}, c_{6}}=$

$$
\int_{x_{0}=0}^{3} \int_{x_{1}=\max \left(x_{0}, 1\right)}^{3}\left[\exp \left(Q_{c_{2}} x_{0}\right)\right]_{1,1} f_{c_{2}}\left(x_{0}, x_{1}\right) d x_{1} d x_{0}
$$

where we have $\left[\exp \left(Q_{c_{2}} x_{0}\right)\right]_{1,1}$ because the subordinated CTMC has to be started in state 1 and has to be in state 1 at the instance when transition $t_{0}$ fires. The limits of the integrals reflect the support of $f_{c_{2}}\left(x_{0}, x_{1}\right)$ and the fact that the firing time of transition $t_{0}$ is smaller than that of transition $t_{1}$. By the same reasoning, we obtain $p_{c_{2}, c_{7}}=$

$$
\int_{x_{0}=0}^{3} \int_{x_{1}=\max \left(x_{0}, 1\right)}^{3}\left[\exp \left(Q_{c_{2}} x_{0}\right)\right]_{1,2} f_{c_{2}}\left(x_{0}, x_{1}\right) d x_{1} d x_{0} .
$$

The probability that transition $t_{1}$ fires first in $P_{0} P_{3} P_{5}$ or in $P_{0} P_{3} P_{6}$ is obtained, respectively, as

$$
\begin{aligned}
& p_{c_{2}, c_{8}}=\int_{x_{1}=1}^{3} \int_{x_{0}=x_{1}}^{4}\left[\exp \left(Q_{c_{2}} x_{1}\right)\right]_{1,1} f_{c_{2}}\left(x_{0}, x_{1}\right) d x_{0} d x_{1}, \\
& p_{c_{2}, c_{9}}=\int_{x_{1}=1}^{3} \int_{x_{0}=x_{1}}^{4}\left[\exp \left(Q_{c_{2}} x_{1}\right)\right]_{1,2} f_{c_{2}}\left(x_{0}, x_{1}\right) d x_{0} d x_{1} .
\end{aligned}
$$

The probability that class 2 is left due to the firing of the EXP transition $t_{b}$ is calculated as

$$
\begin{aligned}
p_{c_{2}, c_{10}}= & \int_{x_{b}=0}^{3} \int_{x_{0}=x_{b}}^{4} \int_{x_{1}=\max \left(x_{b}, 1\right)}^{3} \\
& {\left[\exp \left(Q_{c_{2}} x_{b}\right)\right]_{1,2} \lambda_{b} f_{c_{2}}\left(x_{0}, x_{1}\right) d x_{1} d x_{0} d x_{b} }
\end{aligned}
$$

where $\left[\exp \left(Q_{c_{2}} x_{b}\right)\right]_{1,2} \lambda_{b}$ is the firing intensity of transition $t_{b}$ at time $x_{b}$ and the integration is carried out over all such possible values for which $x_{b} \leq x_{0}$ and $x_{b} \leq x_{1}$.

The calculations for class 3 are very similar to the calculations for class 2. In particular there are two differences to take into account. First, class 3 is reached by the firing of transition $t_{a}$ in marking $P_{0} P_{2} P_{6}$ and not in marking $P_{0} P_{2} P_{5}$ as in case of class 2 . Accordingly, $f_{c_{3}}\left(x_{0}, x_{1}\right)=$

$$
\frac{1}{p_{c_{1}, c_{2}}} f_{t_{1}}\left(x_{1}\right) \int_{x_{a}=0}^{4-x_{0}}\left[\exp \left(Q_{c_{1}} x_{a}\right)\right]_{1,2} \lambda_{a} f_{c_{1}}\left(x_{0}+x_{a}\right) d x_{a} .
$$

with support $0 \leq x_{0} \leq 4,1 \leq x_{1} \leq 3$. Second, while the subordinated CTMCs in class 2 and 3 are identical, in class 3 its initial state is state 2 and not state 1 as in case of class 2 . As class 2, also class 3 has five successors, $c_{i}, 11 \leq i \leq 15$ (see Fig.1). For sake of sparing space we report here only the probability of the transition from class 3 to class 15 :

$$
\begin{aligned}
p_{c_{3}, c_{15}}= & \int_{x_{b}=0}^{3} \int_{x_{0}=x_{b}}^{4} \int_{x_{1}=\max \left(x_{b}, 1\right)}^{3} \\
& {\left[\exp \left(Q_{c_{2}} x_{b}\right)\right]_{2,2} \lambda_{b} f_{c_{3}}\left(x_{0}, x_{1}\right) d x_{1} d x_{0} d x_{b} . }
\end{aligned}
$$

Classes 4 and 5 are easier to handle because there are not enabled GEN transitions in these classes. For both classes the subordinated CTMC is identical to the CTMC of class 1, i.e., $Q_{c_{4}}=Q_{c_{5}}=Q_{c_{1}}$. The difference is that in class 4 the CTMC is started in state 1 while it is started in state 2 in class 5.
Both classes have two successors depending on the marking in which transition $t_{a}$ fires (see Fig.1). We have, for example,

$$
p_{c_{5}, c_{16}}=\int_{x_{a}=0}^{\infty}\left[\exp \left(Q_{c_{5}} x_{a}\right)\right]_{2,1} \lambda_{a} d x_{a},
$$

We omit to describe the construction of the rest of the aggregated graph as it requires similar steps as the ones applied so far. Instead we turn our attention to calculate the steady state probabilities of the markings of the model following the procedure given in Section IV-F.

The first step is to compute the embedded probabilities of the classes. To this end we calculate the steady state probabilities of the DTMC given by the transition probabilities $p_{c_{i}, c_{j}}, 1 \leq i, j \leq 19$. This results in $\left|\pi_{1}^{(e)} \pi_{2}^{(e)} \ldots \pi_{19}^{(e)}\right|=$ $\begin{array}{llllllll}0.25 & 0.1177 & 0.0578 & 0.0415 & 0.0327 & 0.0365 & 0.0125 & 0.0191\end{array}$ $\begin{array}{lllllllll}0.0086 & 0.0412 & 0.0079 & 0.0108 & 0.0032 & 0.0018 & 0.0339 & 0.0390\end{array}$ 0.03520 .18510 .0648 .

The second step is to compute the mean sojourn time for the classes which we illustrate for class 2 . The mean sojourn time is calculated by summing up the mean delays associated with all outgoing events of the class. The result is $S_{2}=$

$$
\begin{aligned}
& \int_{x_{0}=0}^{3} \int_{x_{1}=\max \left(x_{0}, 1\right)}^{3} x_{0}\left[\exp \left(Q_{c_{2}} x_{0}\right)\right]_{1,1} f_{c_{2}}\left(x_{0}, x_{1}\right) d x_{1} d x_{0}+ \\
& \int_{x_{0}=0}^{3} \int_{x_{1}=\max \left(x_{0}, 1\right)}^{3} x_{0}\left[\exp \left(Q_{c_{2}} x_{0}\right)\right]_{1,2} f_{c_{2}}\left(x_{0}, x_{1}\right) d x_{1} d x_{0}+ \\
& \int_{x_{1}=1}^{3} \int_{x_{0}=x_{1}}^{4} x_{1}\left[\exp \left(Q_{c_{2}} x_{1}\right)\right]_{1,1} f_{c_{2}}\left(x_{0}, x_{1}\right) d x_{0} d x_{1}+ \\
& \int_{x_{1}=1}^{3} \int_{x_{0}=x_{1}}^{4} x_{1}\left[\exp \left(Q_{c_{2}} x_{1}\right)\right]_{1,2} f_{c_{2}}\left(x_{0}, x_{1}\right) d x_{0} d x_{1}+ \\
& \int_{x_{b}=0}^{3} \int_{x_{0}=x_{b}}^{4} \int_{x_{1}=\max \left(x_{b}, 1\right)}^{3} x_{b}\left[\exp \left(Q_{c_{2}} x_{b}\right)\right]_{1,2} \lambda_{b} f_{c_{2}}\left(x_{0}, x_{1}\right) d x_{1} d x_{0} d x_{b}
\end{aligned}
$$

Note that the sojourn times are obtained through integrals similar to those used to calculate transition probabilities. Having calculated the sojourn times, $S_{i}, 1 \leq$ $i \leq 19$, the steady state probabilities of the classes are computed by (4). This results in $\left|\pi_{1} \pi_{2} \ldots \pi_{19}\right|=$ $\begin{array}{llllllll}0.1975 & 0.08191 & 0.02360 & 0.04666 & 0.0367 & 0.0210 & 0.0042\end{array}$ $\begin{array}{lllllll}0.0096 & 0.0043 & 0.03044 & 0.00422 & 0.0048 & 0.0013 & 0.0008\end{array}$ $\begin{array}{lllll}0.0250 & 0.0354 & 0.0199 & 0.3684 & 0.0835\end{array}$.

The last step is to distribute the steady state probability of the classes among the involved markings. Once again the procedure is illustrated on class 2 . The probability that both the two GEN transitions have firing time larger than $x, H_{c_{2}}(x)$, is given by $H_{c_{2}}(x)=\int_{x_{1}=\max (x, 1)}^{3} \int_{x_{0}=x}^{4} f_{c_{2}}\left(x_{0}, x_{1}\right) d x_{0} d x_{1}$ if $x \leq 3$ and 0 otherwise.

The proportions according to which class 2 has to be distributed among the markings $P_{0} P_{3} P_{5}$ and $P_{0} P_{3} P_{6}$ is given, respectively, by

$$
\begin{aligned}
& k_{2, P_{0} P_{3} P_{5}}=\int_{0}^{\infty} H_{c_{2}}(x)\left[\exp \left(Q_{c_{2}} x\right)\right]_{1,1} d x, \\
& k_{2, P_{0} P_{3} P_{6}}=\int_{0}^{\infty} H_{c_{2}}(x)\left[\exp \left(Q_{c_{2}} x\right)\right]_{1,2} d x .
\end{aligned}
$$


Having calculated the above proportions for all the classes, the steady state probability of the markings can be obtained. The probability of the markings $P_{0} P_{2} P_{5}, P_{0} P_{2} P_{6}, P_{1} P_{2} P_{5}$, $P_{1} P_{2} P_{6}, \quad P_{0} P_{3} P_{5}, \quad P_{0} P_{3} P_{6}, \quad P_{1} P_{3} P_{5}, P_{1} P_{3} P_{6}, \quad P_{0} P_{4} P_{5}$, $P_{0} P_{4} P_{6}, P_{1} P_{4} P_{5}$ and $P_{1} P_{4} P_{6}$ are $0.1325,0.0649,0.0438$, $0.0396,0.0717,0.0337,0.0581,0.0316,0.0482,0.0233$, 0.2959 and 0.1560 , respectively.

In the following we give a flavour of the numerical quantities involved in the calculations. As mentioned earlier, the transient behaviour of the subordinated CTMCs can be written in closed form. For example, we have $\exp \left(Q_{c_{1}} x\right)=$

$$
\begin{array}{cc}
0 . \dot{0} \dot{3} e^{-2.15 x}\left(16+17 e^{1.65 x}\right) & 0 . \dot{4} \dot{8} e^{-2.15 x}\left(-1+e^{1.65 x}\right) \\
0 . \dot{5} \dot{1} e^{-2.15 x}\left(-1+e^{1.65 x}\right) & 0 . \dot{0} \dot{3} e^{-2.15 x}\left(17+16 e^{1.65 x}\right)
\end{array}
$$

and the other subordinated CTMCs have transient behaviour of similar complexity. The joint pdf of the time to fire values of the GEN transitions in class 2 turns out to be $f_{c_{2}}\left(x_{0}, x_{1}\right)=0.5298\left(0.1353-0.0522 e^{0.5 x_{0}}-\right.$ $\left.1.11510^{-5} e^{2.15 x_{0}}+0.0784 x_{0}\right) x_{1}$ over the support $0 \leq x_{0} \leq$ $4,1 \leq x_{1} \leq 3$. Figure 5 depicts $f_{c_{2}}\left(x_{0}, x_{1}\right)$. In some cases the pdf of the time to fire values is given by different functions in different intervals. For example, the pdf in class 8 is given by the combination of two different functions as

$$
f_{c_{8}}\left(x_{0}\right)= \begin{cases}1.353-0.6906 e^{0.5 x_{0}}- & \text { if } x_{0} \leq 1 \\ 0.0080 e^{2.15 x_{0}}+0.3597 x_{0} & 1.279 \\ 8.535-13.74 e^{0.5 x_{0}}-1.5 e^{2.15 x_{0}}+ \\ e^{2.5 x_{0}}\left(3.53210^{-5}-5.101\right. & \left.10^{-6} x_{0}\right)+ \\ 1.218 x_{0}+ & \text { if } 1 \leq x_{0} \leq 3 \\ e^{0.4 x_{0}}\left(6.051+2.941 x_{0}\right) & \end{cases}
$$

which results in a continuous function depicted in Figure 6.

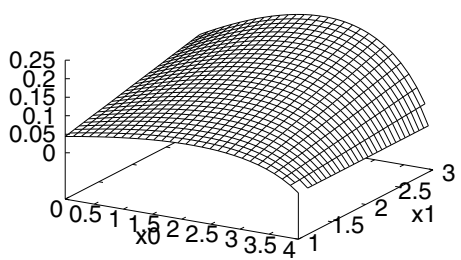

Fig. 5. The joint pdf of the time to fire values in class 2

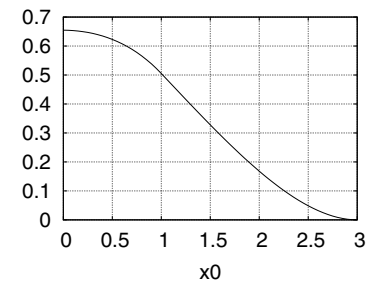

Fig. 6. The pdf of the time to fire value in class 8

\section{DISCUSSION}

In this paper we have presented an extension of the approach based on stochastic state classes. The extension is based on encapsulating into an aggregated stochastic state class those stochastic state classes that share the same situation for what concerns the enabling status of the GEN transitions.

As a practical consequence the extension results in a technique that significantly increases the applicability of the approach based on stochastic state classes. Moreover, the proposed technique can be applied to larger class of nonMarkovian Petri nets than the class of models that are amenable to the analysis through Markov renewal theory [2], [3], or through the method of supplementary variable [1].

The proposed technique results in exact calculus if the transient behaviour of the subordinated CTMCs can be written explicitly. Otherwise, the distributions of the time to fire values can be calculated numerically and fitted then by Bernstein polynomial. This approach results in approximate analysis. The implementation of both cases is underway (while the example presented in this paper has been prepared by hand) and will allow for analysing models with larger state space.

In a more theoretical perspective, the proposed technique makes a step towards the integration of the approach of stochastic state classes [7], [9] with those analytical techniques that already use the concept of separate handling of EXP and GEN transitions [2], [3], [1].

\section{REFERENCES}

[1] R. German and C. Lindemann, "Analysis of Stochastic Petri Nets by the method of supplementary variables," in Performance Evaluation, vol. 20, 1994, pp. 317-335.

[2] G. Ciardo, R. German, and C. Lindemann, "A characterization of the stochastic process underlying a Stochastic Petri Net," IEEE Trans. Softw. Eng., vol. 20, no. 7, pp. 506-515, 1994.

[3] H. Choi, V. G. Kulkarni, and K. Trivedi, "Markov Regenerative Stochastic Petri Nets," Perf. Eval., vol. 20, pp. 337-357, 1994.

[4] C. Lindemann and G.S.Schedler, "Numerical analysis of Deterministic and Stochastic Petri Nets with concurrent deterministic transitions," Performance Evaluation, vol. 27-28, pp. 565-582, 1996.

[5] C. Lindemann and A. Thuemmler, "Transient analysis of Deterministic and Stochastic Petri Nets with concurrent deterministic transitions," Performance Evaluation, vol. 36-37, pp. 35-54, 1999.

[6] M. Telek and A. Horvarth, "Supplementary variable approach applied to the transient analysis of age-MRSPNs," in Proc. Int. Performance and Dependability Symp., 1998, pp. 44-51.

[7] L. Carnevali, L. Sassoli, and E. Vicario, "Using stochastic state classes in quantitative evaluation of dense-time reactive systems," accepted for publication in the IEEE Trans. on Soft. Eng., 2009.

[8] G. Bucci, R.Piovosi, L. Sassoli, and E. Vicario, "Introducing probability within state class analysis of dense time dependent systems," Proc. of the $2^{\text {nd }}$ Int. Conf. on the Quant. Evaluation of Sys.(QEST), September 2005.

[9] L. Sassoli and E. Vicario, "Close form derivation of state-density functions over dbm domains in the analysis of non-Markovian models," Proc. of the $5^{\text {st }}$ Int. Conf. on the Quant. Evaluation of Sys.(QEST), Sept., 2007.

[10] E. Vicario, "Static analysis and dynamic steering of time dependent systems using Time Petri Nets," IEEE Trans. on Soft. Eng., August 2001.

[11] B. Berthomieu and M. Diaz, "Modeling and verification of time dependent systems using Time Petri Nets," IEEE Trans. on Soft. Eng., vol. 17, no. 3, March 1991.

[12] D. Dill, "Timing assumptions and verification of finite-state concurrent systems," Proc.Workshop on Computer Aided Verification Methods for Finite State Systems, 1989.

[13] J. Bengtsson, K. G. Larsen, F. Larsson, P. Pettersson, and W. Yi, UPPAAL: a tool-suite for automatic verification of real-time systems. Hybrid Systems III: LNCS, 1066, 1996.

[14] G. Bucci, L. Sassoli, and E. Vicario, "Oris: a tool for state space analysis of real-time preemptive systems," Proc. of the $1^{\text {st }}$ Int. Conf. on the Quant. Evaluation of Sys.(QEST), 2004.

[15] B. Berthomieu, P.-O. Ribet, and F. Vernadat, "The tool TINA - construction of abstract state spaces for Petri Nets and Time Petri Nets,' International Journal of Production Research, vol. 42, no. 14, 2004.

[16] G. Gardey, D. Lime, M. Magnin, and O. Roux, "Roméo: a tool for analyzing Time Petri Nets," In 17th International Conference on Computer Aided Verification (CAV05),Lecture Notes in Computer Science, 2005.

[17] L. Carnevali, L. Grassi, and E. Vicario, "State-density functions over DBM domains in the analysis of non-Markovian models," IEEE Trans. on Soft. Eng., vol. 35, pp. 178 - 194, March-April 2009.

[18] P.Merlin and D.J.Farber, "Recoverability of communication protocols," IEEE Trans.on Communications, vol. 24, no. 9, 1976.

[19] A. Cumani, "Esp - a package for the evaluation of stochastic Petri nets with phase-type distributed transitions times," in Proc. Int. Workshop on Timed Petri Nets, Torino, Italy, July 1985.

[20] W. J. Stewart, Introduction to the Numerical Solution of Markov Chains. Princeton University Press, 1995. 